\title{
Diferencias por género en la tasa de ganancia salarial de la educación superior y posgrado en México
}

\author{
Marco Antonio Austria-Carlos* \\ Francisco Venegas-Martínez* \\ Gilberto Pérez Lechuga**
}

* Instituto Politécnico Nacional, México
** Universidad Autónoma de Estado de Hidalgo, México

\section{Resumen}

Esta investigación estima la ganancia salarial marginal de la Educación Superior y el Posgrado (ESP) en México con un enfoque de género. Para ello se evalúa el impacto de la ESP sobre los salarios de hombres y mujeres que concluyeron una carrera profesional o un posgrado. La estimación de la tasa de ganancia salarial se realiza mediante el procedimiento propuesto por Heckman, Tobias y Vytlacil (2000) y (2001). La información utilizada para las estimaciones se basa en una muestra de la Encuesta Nacional de Ingresos y Gastos de los Hogares (ENIGH) 2012. El procedimiento de estimación propuesto corrige los problemas asociados con la heterogeneidad y la autoselección de los individuos. El principal resultado, soportado por la evidencia empírica, es que la ESP tiene un impacto positivo sobre los ingresos salariales en ambos géneros, pero el impacto es menor para las mujeres.

Palabras clave: Tasa de ganancia salarial de la educación superior, autoselección, género, México.

\section{Abstract}

Differences by gender in the marginal rate of return to undergraduate and graduate studies in Mexico

This research is aimed at estimating the marginal rate of return to Undergraduate and Graduate Education (UGE) in Mexico with a gender approach. To that end, the impacts of the UGE on the earnings of men and women who have completed an undergraduate or a graduate program are assessed. The estimation of the wage earning rate is carried out by using the procedure proposed by Heckman, Tobias and Vytlacil (2000) and (2001). The information used for the estimates is based on a sample from the National Household Income and Expenditure Survey (ENIGH for its acronym in Spanish) 2012. The proposed estimation procedure addresses the problems associated with heterogeneity and self-selection of individuals. The main empirical result of this research is that UGE has a positive impact on wage income in both genders, but the impact is lower for women.

Keywords: Returns, higher education, self-selection, gender, Mexico. 


\section{INTRODUCCIÓN}

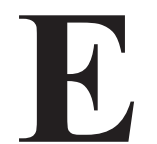

1 diferencial de salarios en la población es un tema de continuo debate y preocupante. Este diferencial en individuos que han concluido una carrera profesional o un posgrado debería reducirse sin importar el género. La presente investigación encuentra evidencia empírica de que la formación académica sí tiene un impacto positivo en la tasa de ganancia salarial (rendimiento) de hombres y mujeres con estudios superiores o de posgrado (maestría o doctorado), pero el impacto es, infortunadamente, menor en el caso de las mujeres. De esta manera, no sólo hay diferencias de oportunidades laborales para las mujeres, sino también en sus salarios.

Con base en la información del Consejo Nacional de Evaluación de la Política de Desarrollo Social (CONEVAL), en México en 2012 existían 53.3 millones de personas en situación de pobreza, de las cuales $41.8 \mathrm{mi}$ llones son pobres moderados y los restantes 11.5 millones se encuentran en situación de pobreza extrema. De los 53.3 millones de mexicanos, 52 por ciento ( 27.6 millones $\left.^{1}\right)$ fueron mujeres, de las cuales alrededor de la mitad tienen una edad menor o igual a 30 años y con familias promedio de 4.14 miembros (superior al promedio nacional ${ }^{2}$ ) y están concentradas en su mayoría en zonas urbanas. ${ }^{3}$

En contraste con lo anterior, como se muestra en la Tabla 1 (renglones 4,5 y 6 ), los indicadores referentes a la población vulnerable por carencias sociales ${ }^{4}$ se presentan en mayor medida en los hombres que en las mujeres tanto en el ámbito urbano como en el rural. Mientras que la población vulnerable por ingresos ${ }^{5}$ se presenta en mayor medida en las mujeres. En el caso de personas que no son pobres ni vulnerables, ${ }^{6}$ la situación se mantiene en las mismas proporciones cuando se desglosa la información por género, pero no así por tamaño de la localidad, ya que la mayoría de la población en estas condiciones se encuentra en el ámbito urbano.

\footnotetext{
${ }^{1}$ De este total, 19 millones se encuentran en el ámbito urbano y 8.6 millones en el ámbito rural.

${ }^{2}$ El promedio nacional, según el INEGI, es de 3.7 miembros en el hogar.

${ }^{3}$ Información sobre otros indicadores puede ser encontrada en Wagstaff (2002).

${ }^{4}$ La vulnerabilidad por carencias sociales se refiere a aquella población que presenta una o más carencias sociales, pero cuyo ingreso es superior a la línea de bienestar.

${ }^{5} \mathrm{La}$ vulnerabilidad por ingresos se refiere a aquella población que no presenta carencias sociales y cuyo ingreso es inferior o igual al de la línea de bienestar.

${ }^{6}$ Aquella población cuyo ingreso es superior a la línea de bienestar y que no tiene carencia social alguna.
} 
Diferencias por género en la tasa de ganancia salarial de la educación superior .../M.A. AUSTRIA CARLOS et al.

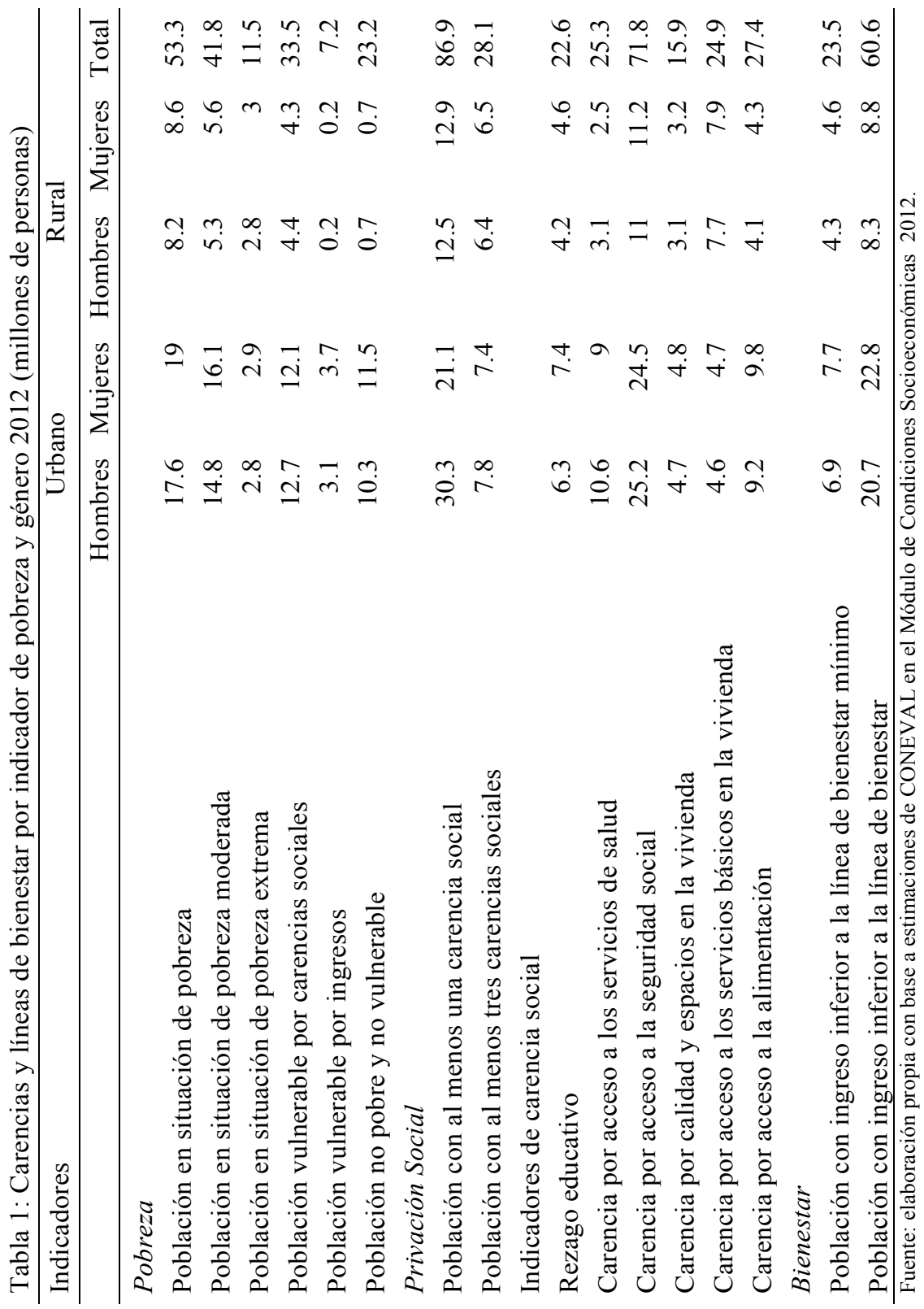


En la Tabla 1 se observa una mayor cantidad de mujeres tanto en zonas urbanas como rurales con rezago educativo, lo cual muestra evidencia de las diferencias existentes en las oportunidades de desarrollo entre hombres y mujeres; situación contraria a los servicios de salud, ya que los hombres son quienes menos concurren a dichos servicios. En lo que respecta a las líneas de bienestar mínimo y $^{7}$ ienestar $^{8}$ se observa, como era de esperarse, que una cantidad mayor de personas en situación de un ingreso inferior a estas líneas de bienestar sean nuevamente mujeres, lo cual demuestra que en México aún se tienen tareas pendientes sobre la equidad de género en materia de salud, educación y alimentación, así como de oportunidades laborales.

Por otro lado, Schultz (1961) considera que la creación de capital humano se sustenta en el planteamiento de que las personas aumentan sus capacidades como productores al invertir en sí mismos y que incrementar el nivel de escolaridad es una inversión deseable. Es importante mencionar en este punto el trabajo de Parker y Pederzini (2000) en el que se destaca que la asistencia escolar de las niñas se reduce después de los 12 años de edad, lo cual tiene un impacto negativo en la formación de capital humano por género. Tomando en cuenta la inequidad ${ }^{9}$ existente en México, el tema de capital humano resulta relevante porque permite explicar en gran medida el desarrollo de los individuos, particularmente los beneficios que ellos reciben de la educación; siendo la tasa de ganancia salarial un indicador importante, ya que ésta impacta directamente en las líneas de bienestar de hombres y mujeres.

La principal contribución de esta investigación es proporcionar evidencia empírica de que la educación tiene un impacto positivo en los ingresos salariales de los individuos de manera diferenciada entre hombres y mujeres, con base en diversos factores observables y subyacentes relacionados con el entorno familiar y económico que impactan de manera significativa la probabilidad de que los individuos decidan estudiar un programa de ESP. Esto permite obtener una tasa de ganancia salarial de acuerdo con la intensidad con que estos factores se presenten por género.

El objetivo de este estudio es estimar el impacto de la ESP en los ingresos de los egresados de una carrera profesional o un posgrado a lo largo de su ciclo de vida considerando el género de los mismos. El marco teórico para analizar las diferencias en el ingreso salarial por género se basa en

\footnotetext{
${ }^{7}$ Equivalente al costo de la canasta alimentaria.

${ }^{8}$ Equivalente a la suma de los costos de la canasta alimentaria y no alimentaria.

${ }^{9}$ Término utilizado en la perspectiva de género para referirse a las diferencias de oportunidades entre mujeres y hombres.
} 
el modelo de capital humano de Mincer (1974). Este modelo provee la base teórica para el estudio de los salarios en función de variables que incrementan la productividad de los individuos. A partir de los trabajos de Schultz (1961) y Becker $(1962,1964)$ se han desarrollado un importante número de contribuciones en la estimación de rendimientos de inversión en capital humano, cuyos resultados demuestran consistentemente que el nivel educativo es un determinante del nivel de ingresos. ${ }^{10}$

Existe una gran cantidad de estudios en muchos países sobre la tasa de ganancia salarial (rendimientos) de la educación, entre los que se encuentran: Hansen (1963), Hanoch (1967), McMahon (1991), Psacharopoulos (1993), Ashenfelter y Krueger (1994), Altonji (1993), Altonji y Dunn (1996), Harmon y Walter (1995), Alba-Ramírez y San Segundo (1995), Cohn y Addison (1998), Card y Ashenfelter (1999), Asplund y Pereira (1999), Card (2000), Harmon, Walter y Westergaard-Nielsen (2001), Carneiro et al. (2001), Psacharopoulos y Patrinos (2002), San Segundo y Valiente (2003), Harmon et al. (2003), Carneiro et al. (2003), Arrazola et al. (2003) y Moffitt (2007). Para el caso de México, existen varias estimaciones de los rendimientos de la educación, por ejemplo: Austria y Venegas-Martínez (2010), Carnoy (1967), Bracho y Zamudio (1994a), Bracho y Zamudio (1994b), Rojas, Angulo y Velásquez (2000), Barceinas (2001), Sarimaña (2002), Del Razo (2003), Rodríguez-Oreggia (2004), López-Acevedo (2004) y Ordaz (2007). En lo que respecta a la literatura relacionada a estudios de género se encuentran: Katrin et al. (2013), Coneval (2012), García (2008), Cabo y Garzón (2007), García y Oliveira (2007), Ariza (2006), Arriagada (2005), CEPAL (2004), CEPAL-UNI FEM (2004), Raczynski (2003), Gómez (2002), Salles y Tuirán (2002), Parker y Pederzini (2000), Gerstenfeld y León (1999), Faúndez (1996), Goldschmidt-Clermont (1995), CEPAL/OREALC (1992) y García de Fanelli (1989), entre muchos otros.

En lo que respecta a los métodos de estimación, Card (1999) en su medición de los rendimientos de la educación encuentra que el estimador de MCO, resultado de una ecuación salarial "Minceriana" (1974), presenta dos sesgos importantes: uno atribuido a la correlación entre la escolaridad y la pendiente de la función de ingresos y el otro relacionado con las habilidades de los individuos, cuyo efecto es capturado en la ordenada al origen de la regresión que se correlaciona con la escolaridad. Ese autor

${ }^{10}$ Para Becker (1964) los gastos en educación o capacitación significan inversiones en capital que concuerdan plenamente con la definición tradicional del concepto de capital (Skipper, 2006). 
incorpora en su metodología econométrica variables instrumentales ${ }^{11}$ para tratar parcialmente ambos sesgos. No obstante, este problema no se resuelve completamente. Para abordar dicha limitación, en este estudio, se emplea el proceso bietápico sugerido por Heckman et al. (2000) y (2001), el cual considera los problemas de sesgo relacionados con la autoselección y la heterogeneidad de los individuos.

De acuerdo con lo anterior, Diez de Medina (1992) menciona en su investigación que el problema de sesgo por autoselección es común en la evaluación del impacto de programas, particularmente en el caso de métodos cuasi-experimentales y surge cuando la extracción de una muestra no se realiza con la misma probabilidad de selección para todos sus elementos, ya sea por la forma de extracción de la muestra o por las características de los individuos bajo estudio, o bien por ambas. En el muestreo aleatorio se cuenta siempre con la información de la probabilidad a priori de extracción de cada individuo en la muestra, por lo que de existir igual probabilidad, una mayor muestra brindará mejores estimaciones. Esto implica que si la muestra no es aleatoria sólo es posible describir características de la muestra, pero no de la población. Diez de Medina (1992) también sugiere que a diferencia del diseño experimental en donde el grupo de control está conformado por personas elegibles que se postulan voluntariamente para recibir un programa o tratamiento, se deberían asignar aleatoriamente a ambos grupos evitando ${ }^{12}$ con ello el problema de sesgo por autoselección; el diseño cuasi-experimental busca conformar un grupo de control a partir de técnicas econométricas con datos externos ${ }^{13}$ al mismo programa. Dado que no existe ningún tipo de asignación aleatoria en la conformación de ambos grupos en el diseño experimental, no es posible asegurar la equivalencia inicial de los grupos de tratamiento y de control, lo que trae como consecuencia, de manera evidente, el problema de sesgo por autoselección y la presencia de posibles problemas de validez tanto interna ${ }^{14}$ como exter$n \mathrm{a}^{15}$ del diseño empleado.

\footnotetext{
${ }^{11}$ Barceinas (2001) menciona que para evitar sesgos en las estimaciones por MCO en el análisis entre escolaridad e ingresos se pueden emplear Variables Instrumentales (VI) donde la condición necesaria para que el método proporcione estimadores consistentes es que los instrumentos utilizados no estén correlacionados con la habilidad del individuo, evitando así el problema de endogeneidad.

${ }^{12}$ Las características observadas y no observadas en el grupo de control y de tratamiento se supone que se distribuyen igual.

${ }^{13}$ Esto se debe a que en su diseño se observan datos existentes que posiblemente su concepción original no tenía como objetivo el de evaluar.

${ }_{14}$ Campbell y Stanley (1973) refieren que el diseño cuasi-experimental tiene validez interna cuando todas las posibles explicaciones quedan controladas por el diseño.

${ }^{15}$ Según Campbell y Stanley (1973) esta validez se refiere a la necesidad de representatividad de las unidades de muestreo, buscando la posibilidad de extrapolar o generalizar los efectos de
} 
Es importante destacar que en la presente investigación no se utilizó otro método de diseño muestral adicional para definir la población objetivo ya que el diseño de la ENIGH es probabilístico, estratificado, unietápico y por conglomerados. De esta manera, a partir de la ENIGH sólo se realizó la extracción de la población de interés. Asimismo, la ENIGH tiene por objetivo obtener información estadística de la distribución y estructura del ingreso y gasto de los hogares, así como de las actividades económicas desarrolladas por los integrantes de los hogares en México. Asimismo, las características de la población objetivo de esta investigación también están incluidas en la representatividad de la ENIGH, por lo que todos individuos en la muestra tienen la misma probabilidad de ser seleccionados. A continuación se resumen los pasos que sigue la investigación:

1. Se parte de la ENIGH.

2. De la ENIGH se extrae la población objetivo.

3. La información extraída contiene variables económicas y características subyacentes de la población objetivo.

4. Estimación de la tasa de ganancia salarial por género a través de la comparación de los grupos de tratamiento y de control.

5. Identificación de los determinantes que inciden en el nivel educativo (tratamiento) y los ingresos salariales (resultado) por género.

Para estimar la tasa de ganancia salarial (rendimientos) de la ESP en México por género se empleará el método cuasi-experimental, ${ }^{16}$ el cual compara los grupos de tratamiento y de control, ${ }^{17}$ siendo posible aislar las características observables de ambos grupos y evaluar en qué medida pueden atribuirse diferencias entre el nivel educativo (tratamiento) y los ingresos salariales (resultado) observados sin incurrir en problemas de sesgo. Se estimará el escenario contrafactual simulando lo que habría ocurrido si el proyecto nunca se hubiera realizado; siendo necesario separar el efecto de las intervenciones de otros factores. En la presente investigación se estiman cuatro efectos de interés, a saber: i) efecto promedio del tratamiento (ATE, por sus siglas en inglés Average Treatment Effect), ii) efecto marginal del tratamiento (MTE, por sus siglas en inglés Marginal Treatment Effect), iii) efecto del tratamiento sobre los tratados (TT, por sus siglas en

los resultados obtenidos a otros contextos tales como: poblaciones, variables de tratamiento y variables de medición.

${ }^{16}$ La ventaja de estos diseños es la existencia de datos, representando menor costo y prontitud en su implementación una vez que el programa ya ha sido desarrollado.

${ }^{17} \mathrm{El}$ grupo de tratamiento representa a aquellos individuos que reciben la intervención; mientras que el grupo de control representa a aquellos que no participan en un programa ni reciben beneficios (Baker, 2000). 
inglés Treatment on the Treated) y iv) efecto promedio del tratamiento local (LATE, por sus siglas en inglés, Local Average Treatment Effect), propuestos en Heckman, Tobias y Vytlacil (2000) y (2001) para la correción del sesgo por autoselección. Para simplificar la notación, en lo sucesivo se hará mención a estos efectos por sus siglas en inglés.

Es necesario enfatizar que en la literatura especializada se evalúa principalmente el impacto de los rendimientos de la educación desde una perspectiva del rendimiento privado. Lo anterior se debe a que la ecuación salarial "Minceriana" (Mincer, 1974) estima rendimientos privados, siendo la forma tradicional de estimar la tasa de rendimiento (TR) de la inversión en educación: La principal limitación de este procedimiento es dejar al margen la estimación de los rendimientos sociales asociados a los gastos (inversión) y otros factores externos asociados al sector público que inciden de manera significativa en los rendimientos de la educación.

Este trabajo se encuentra organizado de la siguiente manera. En la sección 2 se describe el método de estimación de los rendimientos de la Educación Superior. En el transcurso de la sección 3 se describe la muestra empleada y se explican los resultados de las estimaciones econométricas. En la sección 4, se presentan las conclusiones de esta investigación.

\section{MÉTOdo De ESTIMACIóN}

De acuerdo con Baker (2000), en la evaluación de impacto de programas es frecuente emplear técnicas econométricas para inferir sobre el comportamiento de poblaciones que son de interés. También es común que las muestras tengan ciertas cualidades, que de no ser consideradas, pueden hacer suponer que los individuos no deciden llevar a cabo el programa voluntariamente, sino de manera obligada dadas sus características. Considerando esto, el problema de sesgo y heterogeneidad se abordará con la metodología descrita en Heckman et al. (2000) y (2001), misma que se describe a continuación.

Se supone que un individuo obtiene un resultado (salario por hora) con o sin tratamiento (Educación Superior). Para ello, sea $Y_{1}$ el resultado con tratamiento y $Y_{0}$ con ausencia del mismo, por lo que sólo una de estas dos variables se observa para cada individuo. Para evaluar el efecto del tratamiento se debe medir la variable no observable, la cual se obtiene de la diferencia de medias de $Y_{1}-Y_{0}$ con base en las siguientes ecuaciones:

$\mathrm{D}=\mathrm{Z} \theta+\mathrm{U}_{\mathrm{D}}$ 


$$
\begin{gathered}
\mathrm{Y}_{1}=\mathrm{X} \beta_{1}+\mathrm{U}_{1} \\
\mathrm{Y}_{0}=\mathrm{X} \beta_{0}+\mathrm{U}_{0}
\end{gathered}
$$

$$
\left(\begin{array}{l}
U_{D} \\
U_{1} \\
U_{0}
\end{array}\right) \sim N\left(0,\left[\begin{array}{ccc}
1 & \sigma_{1 D} & \sigma_{0 D} \\
\sigma_{1 D} & \sigma_{1}^{2} & \sigma_{10} \\
\sigma_{0 D} & \sigma_{10} & \sigma_{2}^{2}
\end{array}\right]\right)
$$

donde:

La ecuación (1) representa una variable latente ${ }^{18}$ de exposición al tratamiento, y dado que la variable latente, $D$, se encuentra en función del conjunto de variables independientes contenidas en $Z$, aparece de manera natural una restricción de exclusión para el conjunto de variables independientes en $X$ que determinan a $Y_{1}$ y $Y_{0}$. Bajo esta restricción, el mecanismo de elección debe incluir al menos un elemento en $Z$ que no se encuentre en el conjunto $X$; véase, al respecto, Heckman y Vytlacil (2001) ${ }^{19}$ En lo que respecta a las ecuaciones (2) y (3), las variables $Y_{1}$ y $Y_{0}$ representan el logaritmo natural del salario por hora con y sin el programa de ESP, respectivamente. Estas variables dependen, a su vez, de un conjunto de variables contenidas en $X$, los determinantes de la variable dependiente que se observan solo en los dos estados posibles. ${ }^{20}$

Debido a que el impacto de un programa es diferente según los individuos en la muestra, ciertos supuestos condicionarán el tipo de características que se evaluarán en éstos. El primer supuesto, en este estudio, es que el efecto del programa no es igual para todos los individuos. El segundo es que el efecto es diferente entre el grupo de tratamiento y de control, siendo imposible determinar a priori la magnitud de los efectos, y el tercero es que el efecto entre aquellos que deciden llevar a cabo el programa es también diferente, por lo que la decisión de llevar a cabo el programa estará en función de la intensidad con que se presenten sus características observadas y subyacentes. Este último supuesto tiene implicaciones importantes en el diseño de políticas públicas, ya que la focalización eficiente de individuos con determinadas características coadyuvará en el impacto de los programas sobre la población objetivo; véase Heckman y Vytlacil

\footnotetext{
${ }^{18}$ La característica de este tipo de variables es que no pueden ser medidas directamente, pero permiten definir un patrón de respuestas a un grupo de indicadores (Willms, 2006), tal es el caso de los modelos Probit.

${ }^{19}$ Estos autores refieren que el mecanismo de elección es producto de la restricción existente entre las ecuaciones salariales (2) y (3) y la ecuación (1) que contiene la variable latente.

${ }^{20}$ Los estados posibles hacen referencia a que un individuo debe pertenecer a uno y sólo a un grupo en la muestra seleccionada.
} 
(2001). Para estimar los rendimientos de la ESP mediante la ganancia logsalarial esperada (o tasa de ganancia salarial esperada) se estimaran cuatro parámetros propuestos en Heckman et al. (2000) y (2001): ATE, MTE, TT y LATE. Estos se emplean comúnmente para evaluar el impacto de programas y a continuación se explican brevemente sus alcances.

El efecto ATE se define como el rendimiento esperado del programa cuando un individuo es elegido aleatoriamente dentro de la población elegible. ${ }^{21}$ Este parámetro estima la ganancia esperada para cualquier persona dado un conjunto de variables observables contenidas en $X=x$. Para su estimación se emplea la siguiente ecuación:

$\operatorname{ATE}(x)=E\left(Y_{1}-Y_{0} \mid X=x\right)=x\left(\beta_{1}-\beta_{0}\right)$

Este efecto es útil si en lugar de eliminar o reducir un programa se quiere ampliar el programa a toda la población elegible y hacerlo obligatorio para todas las personas que cumplen determinadas características. ${ }^{22}$ Por ejemplo, en materia de programas de desarrollo social, se ha demostrado que estos han traído beneficios sobre la población objetivo, garantizando focalizar la proporción de la población sujeta de recibir el beneficio.

En lo que respecta al MTE, además de estimar las preferencias de los individuos objeto de estudio, estima también el rendimiento esperado del programa para aquéllos individuos que se encuentran en el límite de participar en é $1^{23}$ condicionado al conjunto de variables observables contenidas en $X=x$ y variables subyacentes contenidas en $U_{\mathrm{D}}=u_{\mathrm{D}}$. En este contexto, se desea indagar si los individuos cuentan con variables subyacentes que influyan en la elección de recibir el tratamiento, registrando rendimientos menores. La estimación de este parámetro es formalmente dado por:

$\operatorname{MTE}\left(\mathrm{x}, \mathrm{u}_{\mathrm{D}}\right)=\mathrm{E}\left(\mathrm{Y}_{1}-\mathrm{Y}_{0} \mid \mathrm{X}=\mathrm{x}, \mathrm{U}_{\mathrm{D}}=\mathrm{u}_{\mathrm{D}}\right)=\mathrm{x}\left(\beta_{1}-\beta_{0}\right)+\left(\rho_{1} \sigma_{1}-\rho_{0} \sigma_{0}\right) \mathrm{u}_{\mathrm{D}}$

La utilidad del MTE se desprende de la dependencia que guarda con los valores de $u_{D}{ }^{24}$, de tal manera que si se evalúa el efecto MTE con valores altos de $u_{D}$, se estará calculando la ganancia media para aquellos indivi-

${ }^{21}$ En este estudio, la población elegible está constituida por el grupo de tratamiento y de control. ${ }^{22}$ Heckman (2000) y (2001) afirma que no es útil considerar a toda la población donde se incluyen individuos que no deben ser objetivo del programa, para lo cual es posible restringir el promedio ATE y tomar en cuenta sólo segmentos de población sobre los cuales es importante incidir a través de alguna política pública.

${ }^{23}$ Fue introducido por Björklund y Moffitt (1987), y se trata de la forma límite del parámetro LATE donde los individuos que no han recibido el tratamiento registran un rendimiento ligeramente menor al que pueden acceder si deciden participar en el programa.

${ }^{24}$ Este componente captura todos aquellos factores inobservables que inciden significativamente en la variable latente y que son linealmente independientes de las variables explicativas contenidas en $\mathrm{Z}$. 
duos cuyos factores inobservables hacen menos probable su participación en el tratamiento, y lo contrario para valores bajos de $u_{D}$. Así, si $u_{D}=0$, entonces el MTE es igual al parámetro ATE. ${ }^{25} \mathrm{Si}$ bien el efecto MTE resulta ser la forma límite del efecto LATE, este efecto suele ser una herramienta útil para demostrar la existencia de externalidades que suelen afectar la probabilidad de que los individuos decidan llevar o no el programa; de ahí el signo negativo esperado y que en el caso de programas sociales puede significar su éxito o fracaso.

En lo que respecta al efecto TT, éste se define como el rendimiento esperado del programa para aquellos individuos que eligieron participar y que en realidad recibieron el tratamiento voluntariamente. Por lo que la ganancia esperada de quienes efectivamente han recibido el tratamiento $(\mathrm{D}=1)$ sujeto al conjunto de variables observables contenidas en $X=x, \mathrm{y}$ $\mathrm{Z}=\mathrm{z}$ está dada por:

$\mathrm{TT}(\mathrm{x}, \mathrm{z}, \mathrm{D}=1)=\mathrm{E}(\mathrm{Y} 1-\mathrm{Y} 0 \mid \mathrm{X}=\mathrm{x}, \mathrm{Z}=\mathrm{z}, \mathrm{D}=1)=\mathrm{x}\left(\beta_{1}-\beta_{0}\right)+\left(\rho_{1} \sigma_{1}-\rho_{0} \sigma_{0}\right)$ $(\varphi(\mathrm{z} \theta / \Phi(\mathrm{z} \theta))$

donde $\varphi(\cdot)$ representa la función de densidad de una variable aleatoria normal estándar, $\Phi(\cdot)$ denota su función de distribución acumulada, $r_{1}$ y $r_{0}$ son los coeficientes de correlación entre $U_{1}$ y $U_{\mathrm{D}}, \mathrm{y} U_{0} \mathrm{y} U_{\mathrm{D}}$, respectivamente. La relevancia de este efecto consiste en demostrar que el programa registra el impacto esperado sobre los ingresos de los individuos que decidieron voluntariamente llevar a cabo el programa y que además es mayor en comparación con aquellos con un menor nivel educativo.

El efecto $\mathrm{LATE}^{26}$ se define como el rendimiento esperado del programa debido a cambios en los factores observables contenidos en $Z_{\mathrm{k}}$, los cuales inducen a los individuos a recibir el programa. Este efecto se define a partir de un cambio de $Z \mathrm{q}=z$ a $Z \mathrm{q}=z$ 'q con $z \mathrm{q}<z$ 'q, siendo $z$ y $z$ ' iguales en todo, excepto en el $k$-ésimo elemento. Para estimar este parámetro, formalmente, se escribe:

$$
\begin{aligned}
& \operatorname{LATE}\left(D(z)=0, D\left(z^{\prime}\right)=1, X=x\right)=E\left(Y_{1}-Y_{0} \mid D(z)=0, D\left(z^{\prime}\right)=1, X=x\right) \\
& =x\left(\beta_{1}-\beta_{0}\right)+\left(\rho_{1} \sigma_{1}-\rho_{0} \sigma_{0}\right)\left(\varphi\left(z^{\prime} \theta\right)-\varphi(z \theta) / \Phi\left(z^{\prime} \theta\right)-\Phi(z \theta)\right)
\end{aligned}
$$

La relevancia de este efecto es que permite simular impactos esperados (efectos locales) del programa, producto de variaciones en los valores de

\footnotetext{
${ }^{25}$ En la medida que $u_{\mathrm{D}}$ se aproxima a cero, resulta más probable que los individuos decidan llevar a cabo el programa, ya que no existen factores subyacentes que impidan llevar a cabo un programa de ES.

${ }^{26}$ Como una versión del MTE, el efecto LATE fue introducido por Imbens y Angrist (1994), y la forma límite del LATE se debe a Heckman (1997) y Angrist et al. (2000).
} 
variables de interés, que al ser comparadas directamente con los resultados del efecto ATE permiten plantear y definir estrategias que coadyuven al logro de los objetivos del programa de manera eficiente. ${ }^{27}$ En esta investigación, los efectos LATE ayudan a simular los rendimientos esperados de los individuos cuando se hace variar la educación de los padres y el tamaño del hogar, siendo útil para estimar la magnitud del impacto de las estrategias de interés.

A continuación se presentan los resultados obtenidos con los cuatro parámetros de interés empleando el proceso bietápico propuesto en Heckman et al. (2000) y (2001). En la primera etapa se estima un modelo Probit donde se calcula la variable auxiliar $l$, la cual captura el efecto del sesgo de autoselección presente en la variable latente $D$. En la segunda etapa se estiman las ecuaciones salariales "Mincerianas" empleando las variables auxiliare $l$ para corregir el sesgo originado por el truncamiento de las variables $Y_{1}$ y $Y_{0}$, permitiendo con ello obtener parámetros confiables con énfasis en el enfoque de género.

\section{ESTIMACIÓN Y RESULTADOS EMPÍRICOS}

A continuación se muestran las estimaciones obtenidas a partir de las ecuaciones (1) a (3), describiendo primero las características de la muestra empleada.

\section{La muestra}

Para el análisis econométrico se emplea información de la Encuesta Nacional de Ingresos y Gastos de los Hogares (ENIGH, 2012), la cual contiene información sobre diferentes características socioeconómicas de los individuos. ${ }^{28}$ La restricción más importante en relación a la información disponible en la encuesta es que no incluye toda la información sobre todas las características familiares empleadas en este estudio; por ejemplo, no incluye datos sobre el tamaño del hogar, nivel de ingreso de la familia y experiencia que son importantes para explicar la elección de los individuos (hijos) de ingresar o no a un programa de ESP, siendo necesario construir las variables de la manera tradicional a partir de la información disponible.

\footnotetext{
${ }^{27}$ Este parámetro se estima, generalmente sobre las variables determinantes en la decisión de que los individuos lleven a cabo o no el programa.

${ }^{28}$ Se empleó el Software Stata (versión 12).
} 
La construcción de la muestra ${ }^{29}$ se realizó bajo las siguientes consideraciones $^{30}$ :

1. Se incluyó a hombres y mujeres que vivieran con sus padres, contando así con el nivel educativo e ingreso del jefe del hogar. ${ }^{31}$

2. Se eliminaron aquellos individuos cuyo ingreso del jefe del hogar no estuviera especificado (missing values), incluyendo a hijos que tuvieran un sueldo positivo.

3. La edad de los individuos se acotó en el rango de 22 a 65 años. ${ }^{32}$

4. Se consideró a los asalariados que trabajaran más de 20 horas a la semana. ${ }^{33}$

5. No se distinguió entre individuos que estudiaron en escuelas públicas y/o privadas.

6. No se consideraron los ingresos provenientes de actividades diferentes al mes pasado y actividades realizadas antes de los seis meses al levantamiento de la encuesta. ${ }^{34}$

Bajo los supuestos anteriores, se obtuvo una muestra de 7150 individuos. Las siguientes variables para estimar en rendimiento del programa de educación fueron utilizadas:

1. Treatment: variable de tratamiento que toma el valor de 1 cuando el individuo cuenta con programa de ESP y 0 cualquier otro caso. ${ }^{35}$

2. Lsalario: logaritmo natural del salario por hora de los hijos.

${ }^{29}$ Es relevante destacar que no se empleó ningún método de diseño muestral adicional para definir nuestra población objetivo; esto derivado de que el diseño de la ENIGH es probabilístico, estratificado, unietápico y por conglomerados. En ese sentido, a partir de la ENIGH solo se realizó una extracción de la población de interés.

${ }^{30}$ En virtud de que el objetivo de la ENIGH es obtener información estadística de la distribución, monto y estructura del ingreso y gasto de los hogares, así como las actividades económicas desarrolladas por los miembros de los hogares en México. Las características de la población objetivo de esta investigación también están incluidas en la representatividad de la ENIGH, por lo que nuestra muestra tiene la misma probabilidad de ser seleccionada.

${ }^{31}$ Es posible inferir sobre el papel de los padres, tanto social como económico, en la decisión de que los hijos lleven a cabo un programa de ESP y cómo éste afecta los ingresos de los hijos.

${ }^{32}$ Se eliminaron de la muestra a individuos en las edades de 0 a 12 años que hayan registrado ingresos por trabajo principal. Además, a partir de los 22 años, en promedio, los individuos egresan de la universidad.

${ }^{33} \mathrm{Se}$ descartan ingresos asociados a algún ingreso distinto del salario, por ejemplo, una beca.

${ }^{34}$ El objetivo es contar con los ingresos relacionados a las actividades laborales prevalecientes al momento del levantamiento de la encuesta, teniendo condiciones de análisis equitativas y comparables.

${ }^{35}$ Por los años acumulados de educación formal reportados se asignó "1" al grupo de tratamiento cuando el número de años de educación se ubico en los siguientes niveles: Normal (16 años), Carrera Técnica o Comercial (15 años), Profesional (18 años), Maestría y Doctorado (20 a 23 años, respectivamente). Se asignó " 0 " a aquellos individuos cuyo número de años de educación se ubico en los siguientes niveles: sin educación (cero años), Preescolar (un año), Primaria (seis años), Secundaria (tres años), Preparatoria o Bachillerato (tres años). 
3. Tam_hogar: número de miembros en el hogar (incluye total de integrantes, huéspedes y trabajadores domésticos). ${ }^{36}$

4. Educación_padre: número de años acumulados de educación del jefe de familia. ${ }^{37}$

5. Educación madre: número de años acumulados de educación de la madre de familia.

6. Lsalario jefe: logaritmo natural del salario por hora del jefe del hogar.

7. Edad: número de años cumplidos por los hijos al momento de la entrevista.

8. Exper: número de años de experiencia laboral. ${ }^{38}$

9. Exper2: cuadrado de la variable experiencia. ${ }^{39}$

10. Género: variable binaria donde 1 es mujer y 0 es hombre.

Con base en los supuestos y las variables consideradas, a continuación se muestran las estimaciones de los rendimientos de la ESP mediante los cuatro parámetros anteriormente definidos.

\section{Resultados empíricos}

Para analizar la composición de la población en la muestra empleada por grupos de edad, en la Figura 1, se observa que 51 por ciento del total de los individuos en la muestra se concentran en el rango de edad de 22 a 26 años de edad y en los tres primeros quintiles. Como era de esperarse, en el grupo de tratamiento, conforme la edad se incrementa también lo hacen los ingresos gradualmente y comienzan a reducirse conforme logran rebasar la barrera del rango de edad que va de 47 a 51 años de edad. En el caso del grupo de control dicha caída en el ingreso comenzó a partir del rango de edad de 37 a 41 años de edad, lo cual muestra que los individuos que no deciden adoptar un programa de ESP a lo largo de su ciclo de vida son más propensos a presentar carencias sociales en un periodo de tiempo más

\footnotetext{
${ }^{36}$ La evidencia demuestra que los hogares grandes registran un impacto negativo en el nivel de bienestar de sus integrantes, ya que el ingreso tiende a redistribuirse entre un mayor número de integrantes (Alonzo, 2004).

${ }^{37}$ Con el nivel de instrucción reportado en la ENIGH se especificó el número de años acumulados con que contaban los padres, los cuales se encuentran entre 0 y hasta 23 años de educación formal.

${ }^{38}$ En virtud de que la ENIGH no contiene información sobre experiencia laboral, ésta se construyó del modo usual: Exper $=$ Edad —años de educación formal — 6 .

${ }^{39}$ Se desea mostrar la existencia de rendimientos decrecientes. En vista de que el capital humano está relacionado con la productividad, una productividad marginal constante contradice el supuesto que subyace en la ley de los rendimientos decrecientes. Diez de Medina (1992) estima ecuaciones salariales, encontrando los signos esperados, positivos para la variable Experiencia y negativo para la variable Experiencia al Cuadrado.
} 
corto, o algún tipo de vulnerabilidad ya sea social o de ingresos, incluso pueden llegar a presentar algún tipo de situación de pobreza.

Figura 1: Distribución de individuos por grupo de edad y quintiles

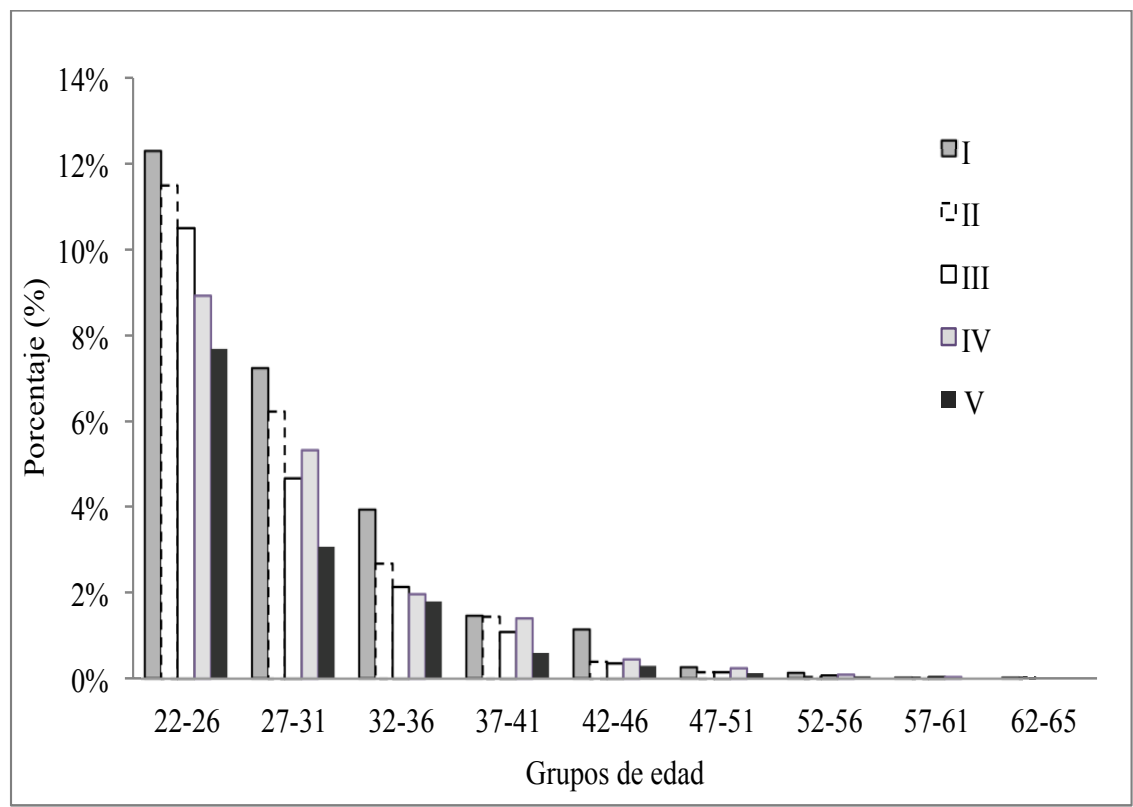

Fuente: elaboración propia con datos de la ENIGH 2012.

En la Tabla 2 se muestran las estadísticas descriptivas para el grupo de tratamiento y de control, respectivamente. Los datos sugieren la existencia de diferencias salariales importantes entre los grupos, aun cuando la edad promedio es similar. Es también posible observar que la escolaridad promedio de los padres es mayor en el grupo de tratamiento; mientras que el tamaño del hogar y la proporción de mujeres es mayor en el grupo de control. También se observa que las mujeres cuentan con un menor ingreso en ambos grupos, lo que muestra brechas de inequidad de la distribución de ingreso siendo que las mujeres cuentan con el mismo nivel educativo que los hombres pero con trabajos mal remunerados. En este sentido, la información sugiere que las especificaciones econométricas requieren un tratamiento apropiado que evite incurrir en posibles sesgos en los estimadores.

Una vez identificada la heterogeneidad entre ambos grupos se procede a estimar el rendimiento de la ESP sobre los salarios de los hijos, es necesario corregir el sesgo por autoselección mediante una técnica biétapica, la cual requiere de un modelo Probit en la primera etapa y en la segunda una ecuación salarial "Minceriana". 
Tabla 2: Estadísticas descriptivas de los grupos de tratamiento y de control

\begin{tabular}{lrrrrr}
\hline Variables & Observaciones & Promedio & $\begin{array}{r}\text { Desviación } \\
\text { Estándar }\end{array}$ & Mínimo & Máximo \\
\hline Grupo de Tratamiento & & & & & \\
Tam_Hog_2 & 2546 & 4.71 & 1.72 & 2 & 14 \\
Educación_padre & 2546 & 9.86 & 6.6 & 0 & 23 \\
Educación_madre & 2546 & 10.84 & 4.94 & 0 & 23 \\
Edad & 2546 & 27.87 & 5.47 & 22 & 59 \\
Exper & 2546 & 4.45 & 5.4 & 0 & 38 \\
Lsalario & 2546 & 3.35 & 0.87 & -0.72 & 6.25 \\
Lsalario Mujeres & 1283 & 3.31 & 0.88 & -0.72 & 5.57 \\
Lsalarios Hombres & 1283 & 3.38 & 0.87 & -0.62 & 6.25 \\
Grupo de Control & & & & & \\
Tam_Hog_2 & 4604 & 5.56 & 2.4 & 2 & 21 \\
Educación_padre & 4604 & 5.98 & 4.7 & 0 & 20 \\
Educación_madre & 4604 & 6.93 & 4.12 & 0 & 23 \\
Edad & 4604 & 27.27 & 6.36 & 22 & 64 \\
Exper & 4604 & 11.89 & 7.35 & 3 & 54 \\
Lsalario & 4604 & 2.68 & 0.85 & -3.33 & 5.51 \\
Lsalario Mujeres & 1583 & 2.61 & 0.86 & -3.33 & 5.05 \\
Lsalarios Hombres & 3021 & 2.72 & 0.86 & -3.10 & 5.51 \\
\hline Find
\end{tabular}

Fuente: elaboración propia con datos de la ENIGH 2012.

Derivado de lo anterior, en la Tabla 3 se muestra la estimación del modelo Probit, cuya variable dependiente está constituida por 2546 individuos con ESP y 4604 con un nivel educativo menor; las variables empleadas como mecanismo de elección ${ }^{40}$ son el ingreso del jefe de familia y el tamaño del hogar. En el modelo econométrico, se observa que los hijos tienen mayor probabilidad de adoptar el programa de Educación Superior en la medida que sus padres tengan mayor nivel educativo, ${ }^{41}$ mayor ingreso ${ }^{42}$

\footnotetext{
${ }^{40}$ Pueden haber otros factores que afecten la decisión de los hijos a adoptar o no el programa; sin embargo, se eligieron estos mecanismos porque podrían impactar en la decisión de adoptar o no un programa.

${ }^{41}$ Dado que el nivel educativo permite a los individuos desarrollar más y mejores habilidades que impactan en su calidad de vida, los padres con un nivel educativo relativamente alto incentivaran a sus hijos a contar con mayor número de años de educación formal con el objetivo de que estos gocen de un mayor bienestar social; igual e inclusive mejor al de los padres a lo largo del ciclo de vida laboral de los individuos.

${ }^{42}$ Cuando existe pobreza por ingresos se ha demostrado que aumenta la probabilidad de que un importante sector de la población no pueda adquirir la canasta de bienes no alimentarios como
} 
y donde el tamaño del hogar ${ }^{43}$ tiende a ser reducido. Cabe destacar el signo del coeficiente de la variable género, ${ }^{44}$ el cual demuestra que las mujeres tienen mayor probabilidad de adoptar un programa de Educación Superior, a pesar de que son ellas quienes más registran carencias sociales en materia educativa, vivienda y alimentación, así como situación de pobreza.

Tabla 3: Modelo Probit para el Programa de Educación Superior ${ }^{\mathrm{a}}$

\begin{tabular}{lrl}
\hline Variables & Coeficientes & $\mathrm{P}>(\mathrm{z})$ \\
\hline Constante & -0.260 & $(0.00)^{*}$ \\
Educación_madre & 0.047 & $(0.00)^{*}$ \\
Educación_padre & 0.037 & $(0.00)^{*}$ \\
Exper & -0.211 & $(0.00)^{*}$ \\
Exper2 & 0.005 & $(0.00)^{*}$ \\
Lsalario_jefe & 0.217 & $(0.00)^{*}$ \\
Tam_Hogar & -0.098 & $(0.00)^{*}$ \\
Género & 0.452 & $(0.01)^{*}$ \\
\hline
\end{tabular}

${ }^{\mathrm{a}}$ Variable dependiente: treatment (tratamiento y control)

* $P(z)$ significativo al $5 \%$.

Fuente: elaboración propia con datos de ENIGH 2012.

A partir de los resultados del modelo Probit se estimó la variable auxiliar, $l$, la cual mide el efecto por autoselección y corrige el sesgo en que se incurre al estimar las ecuaciones salariales por MCO. En este sentido, la Tabla 4 muestra la ecuación salarial "Minceriana" para los grupos de tratamiento y control. El propósito de emplear ambos grupos en la estimación es destacar el papel de la variable $l$. Dada su significancia estadística, es posible deducir que la heterogeneidad y la autoselección influyen de manera importante en la diferencia salarial entre ambos grupos. A partir de la Tabla 4 se destaca el valor del coeficiente de la variable género, el cual demuestra que las mujeres tienden a contar con ingreso menor en promedio que los hombres, por lo que existe evidencia suficiente en esta etapa del estudio para demostrar que la inequidad en materia de género es un hecho real en términos del ingreso que perciben las mujeres aun cuando ellas cuentan inclusive con el mismo nivel educativo que los hombres.

la educación.

${ }^{43}$ En hogares con altos niveles de marginación se ha observado que el número de miembros suele ser generalmente mayor en comparación con aquellos con menor nivel de marginación (Alonzo, 2004).

${ }^{44}$ Esta situación puede deberse en gran medida a que en el grupo de tratamiento existe una proporción mayor de mujeres; sin embargo, en términos del ingreso percibido la situación cambia radicalmente. 
Tabla 4: Ecuación salarial minceriana ${ }^{\mathrm{a}}$

\begin{tabular}{lrr}
\hline \multicolumn{1}{c}{ Variables } & Coeficientes & $\mathrm{P}>\mathrm{z}$ \\
\hline Constante & 4.460 & $(0.00)^{*}$ \\
Educación_padre & 0.009 & $(0.00)^{*}$ \\
Educación_madre & -0.002 & $(0.35)$ \\
Exper & 0.120 & $(0.00)^{*}$ \\
Exper2 & -0.003 & $(0.00)^{*}$ \\
Género & -0.231 & $(0.00)^{*}$ \\
Lambda & -3.706 & $(0.00)^{*}$ \\
\hline${ }^{a}$ Variable dependiente: Lsalario (logaritmo natural del salario). & \\
${ }^{*} P(t)$ significativo al 5\%. & \\
Fuente: elaboración propia con datos de ENIGH 2012. &
\end{tabular}

Adicionalmente, en la Tabla 5 se estiman las ecuaciones salariales para el grupo de tratamiento y de control, respectivamente, considerando el efecto del sesgo por autoselección mediante la variable auxiliar I . En este caso, las ecuaciones salariales miden el efecto que tiene las variables independientes consideradas sobre los ingresos salariales de los hijos. En ambas estimaciones la variable auxiliar es estadísticamente significativa, lo que expresa que existe suficiente evidencia para afirmar que de no controlarse la heterogeneidad y la autoselección, los estimadores por MCO estarían sesgados. De la Tabla 5 también se destaca el signo esperado de los coeficientes estimados; positivos para la variable experiencia y negativos para la variable experiencia al cuadrado. Por otra parte se observa que únicamente la educación de la madre en el grupo de control fue estadísticamente significativa, evidenciando que mientras ésta cuente con un mayor nivel educativo se incrementará la probabilidad de que los hijos elijan llevar a cabo un programa de ESP. En el enfoque del modelo minceriano, la educación de los padres en el grupo de tratamiento no fueron significativos, dejando entrever que no hay suficiente evidencia para ver cómo impacta la educación de estos sobre el nivel de ingreso de los hijos. No obstante, es factible suponer que de incrementarse el nivel educativo de los padres mediante diversas estrategias de política pública, una proporción mayor de individuos (hijos) que se encuentran en la edad límite podrían adoptar el programa potencializando así sus capacidades y habilidades.

En la Tabla 5 se destaca el signo negativo de la variable género, lo que demuestra que existe evidencia suficiente sobre la inequidad existente entre hombres y mujeres, ya que éstas últimas en promedio perciben un menor nivel de ingresos en ambos grupos. 
Tabla 5: Ecuación salarial para los grupos de tratamiento y de control $^{\mathrm{a}}$

\begin{tabular}{lrr}
\hline Variables & Regresión múltiple & $\mathrm{P}>(\mathrm{t})$ \\
\hline Grupo de Tratamiento & & $(0.00)^{*}$ \\
Exper & 0.171 & $(0.00)^{*}$ \\
Exper2 & -0.004 & $(0.71)$ \\
Educación_padre & -0.001 & $(0.94)$ \\
Educación_madre & 0.000 & $(0.00)^{*}$ \\
Género & -0.227 & $(0.00)^{*}$ \\
Lambda & -2.979 & $(0.00)^{*}$ \\
Constante & 4.256 & \\
Grupo de Control & & $(0.00)^{*}$ \\
Exper & 0.082 & $(0.00)^{*}$ \\
Exper2 & -0.002 & $(0.16)$ \\
Educación_padre & -0.004 & $(0.00)^{*}$ \\
Educación_madre & 0.014 & $(0.00)^{*}$ \\
Género & -0.233 & $(0.00)^{*}$ \\
Lambda & -2.464 & $(0.00)^{*}$ \\
Constante & 3.760 &
\end{tabular}

${ }^{a}$ Variable dependiente: Lsalario (logaritmo natural del salario).

* $P(t)$ significativo al $5 \%$.

Fuente: elaboración propia con datos de ENIGH 2012.

Por otra parte, para calcular el efecto promedio de la ESP en México en la muestra empleada, a partir de las ecuaciones salariales y su diferencia de medias, se calculó el ATE. Para la estimación del MTE se empleó el diferencial existente entre las variables auxiliares $l$. Para el efecto TT, se emplearon de manera combinada los resultados obtenidos del ATE y el MTE; mientras que para el LATE se emplearon los efectos ATE y MTE, así como los cambios marginales de los mecanismos de elección definidos en el apartado "Resultados empíricos" de esta investigación.

La Tabla 6 registra los efectos estimados del tratamiento para el grupo de individuos considerados en la muestra haciendo una especial distinción entre el género de los mismos. El cuadro muestra que el rendimiento promedio (ATE) por un año ${ }^{45}$ adicional de Educación Superior es aproximadamente de 11.2 por ciento para un individuo que es tomado aleatoriamente

${ }^{45}$ Los efectos porcentuales se calculan escalando el efecto total entre la diferencia promedio de años de educación escolar que existe entre el grupo de tratamiento y de control (7.2747 años). 
de la población, mientras que el rendimiento para un individuo que efectivamente ha recibido el tratamiento (TT) es equivalente a 9.53 por ciento. Ambos resultados sugieren que la implementación de un programa de Educación Superior trae consigo rendimientos positivos y en promedio más altos en comparación a aquellos individuos con un menor nivel educativo. En lo que respecta al efecto por género, los rendimientos del programa son siempre mayores para los hombres tanto en el ATE como en el TT; pero además los rendimientos para los hombres están por encima del ATE (11.2 por ciento) y el TT ( 9.53 por ciento), mientras que el rendimiento para las mujeres se encuentra por debajo de dichos promedios, respectivamente.

Tabla 6: Efectos del programa de Educación Superior sobre los ingresos de los individuos en México

\begin{tabular}{lr}
\hline Efectos & Valor (\%) \\
\hline Rendimiento promedio del tratamiento (ATE) & 11.20 \\
Mujeres & 11.12 \\
Hombres & 11.25 \\
Marginal Treatment Effect (MTE) & -0.064 \\
Tratamiento de los tratados & 9.53 \\
Mujeres & 9.50 \\
Hombres & 9.54 \\
\hline
\end{tabular}

Fuente: elaboración propia con datos de ENIGH 2012.

El signo negativo del efecto marginal del tratamiento (MTE) demuestra que los individuos en la muestra cuentan con variables no observables (externalidades) contenidas en $u_{\mathrm{D}}$ que pesan significativamente sobre la elección de recibir el tratamiento, registrando rendimientos menores como resultado de no participar en el programa; véase al respecto Heckman (2005). Por ejemplo, en el caso particular de las mujeres, quienes cuentan con mayor rezago educativo, es deseable identificar en otros estudios aquellas externalidades que logren explicar significativamente la inequidad entre hombres y mujeres en materia educativa, salud e ingresos, entre otros aspectos relacionados con el desarrollo social.

Para complementar el análisis, en la Tabla 7 se muestra el rendimiento esperado del programa como resultado de cambios en los factores observables contenidos en $Z_{\mathrm{k}}{ }^{46}$ Se determinó la magnitud del impacto sobre el efecto promedio (ATE) cuando se produce un cambio marginal en el número de años de educación formal de los padres y en el tamaño del hogar.

\footnotetext{
${ }^{46}$ Estos resultados se construyen a partir del modelo Probit.
} 
De este cuadro también se desprende que si la educación de la madre se incrementara marginalmente, además de ampliar la probabilidad de que el hijo decida participar en el programa de ESP, se impactaría positivamente sobre los rendimientos marginales del salario del hijo en 0.04 puntos porcentuales más, pasando de 11.2 por ciento del efecto ATE a 11.235 por ciento en el efecto local.

Tabla 7: Efecto Local Promedio del Tratamiento (LATE)

\begin{tabular}{lr}
\hline Efectos & Valor (\%) \\
\hline Educación de la madre & 11.235 \\
Educación del padre & 11.227 \\
Tamaño del Hogar & 11.114 \\
\hline
\end{tabular}

Fuente: elaboración propia con datos de ENIGH 2012.

En el caso de la educación del padre, un año más en la educación formal de éste reflejaría un rendimiento marginal positivo del programa sobre el salario del hijo en 0.03 puntos porcentuales más, pasando de 11.2 por ciento del efecto ATE a 11.227 por ciento en el efecto local. Como era de esperarse, el impacto de la educación de la madre resultó ser ligeramente mayor a la del padre ${ }^{47}$. Otro efecto local que interesa evaluar es el relacionado con el tamaño del hogar. A partir del modelo Probit, se tiene que la probabilidad de que un individuo decida participar en un programa de ESP está condicionado, entre otras variables, al número de integrantes en la familia, por lo que en la medida de que este número se incremente se impactará negativamente en los rendimientos de la ESP de los salarios de los hijos. En este sentido, se tiene que cuando se incrementa el tamaño del hogar marginalmente, se impacta negativamente sobre los rendimientos de la ESP en 0.08 puntos porcentuales menos, pasando de 11.2 por ciento del efecto ATE a 11.11 por ciento en el efecto local.

\section{Conclusiones}

Con base en Heckman et al. (2000) y (2001) y el análisis de género se han estimado cuatro efectos relevantes para medir los rendimientos de la ESP

${ }^{47}$ A pesar de que las mujeres cuentan con el mayor número de carencias sociales y situación de pobreza, son ellas quienes tienen mayor injerencia sobre los rendimientos de los ingresos de los hijos. Al respecto Jiménez-Villacís (2008) menciona que a la mujer le han sido asignados culturalmente diversos papeles a desarrollar en la sociedad, cuyas formas sociales estas alineadas a su rol como madres, esposas, amas de casa, enfermeras y educadoras, entre otros. Les han atribuido otros roles como el biológico que resulta en la responsabilidad de brindar afecto a los hijos basado en las características funcionales como la maternidad y tareas del hogar relacionadas con las actividades de los hijos, lo que le ha permitido culturalmente tener mayor acercamiento y manifestaciones de afecto, las que influyen en su comportamiento, motivación y actitudes en el ambiente escolar. 
en México y su efecto sobre los ingresos de los individuos mediante un modelo Heckit. Los efectos estimados consideran la heterogeneidad y el sesgo por autoselección persistentes en la muestra obtenida a partir de la ENIGH 2012.

Los datos descriptivos mostraron la heterogeneidad entre el grupo de tratamiento y control, tales como diferencias salariales significativas, escolaridad mayor en los padres del grupo de tratamiento y mayor tamaño del hogar en el grupo de control. También se encontró en este análisis que las mujeres cuentan con un menor ingreso salarial en ambos grupos, evidenciando inequidad en la distribución de ingreso en México en aquellos casos en que las mujeres contaban con el mismo nivel educativo que los hombres. Asimismo, los resultados obtenidos indican que el rendimiento de la ESP para un individuo en México es positivo y mayor respecto a aquellos que cuentan con un menor nivel educativo. Se demostró que si un individuo es tomado aleatoriamente de la población elegible, el rendimiento alcanzado (ATE) es mayor en comparación a aquellos que efectivamente recibieron el programa de Educación Superior (TT).

Con respecto al parámetro ATE, se estimó un rendimiento promedio por año adicional de ESP de 11.2 por ciento para un individuo que es tomado aleatoriamente de la población; mientras que para el efecto TT se estimó un rendimiento de 9.53 por ciento para un individuo que efectivamente haya recibido el tratamiento de manera voluntaria. Ambos resultados muestran que la implementación de un programa de ESP trae consigo rendimientos positivos y en promedio más altos en comparación a aquellos individuos con un menor nivel educativo. En lo que respecta al efecto por género, los rendimientos del programa fueron siempre mayores para los hombres tanto en el ATE como en el TT, pero además dichos rendimientos para los hombres estuvieron por encima del ATE (11.2 por ciento) y el TT (9.53 por ciento), mientras que el rendimiento para las mujeres se encuentró por debajo de dichos promedios, respectivamente. Esto extiende los resultados encontrados en los estudios de García de Fanelli (1989) y Ariza (2006) sobre discriminación salarial por género. Los resultados sugieren que es factible ampliar la población elegible del programa de ESP en lugar de reducirla, porque existen individuos con las características suficientes para ser incorporados, lo que permitiría a lo largo de su ciclo de vida incentivar sus ingresos y con ello ampliar la gama de posibilidades en el gasto de la canasta alimentaria y no alimentaria; y de manera particular en las mujeres, quienes cuentan con menor nivel de rendimiento. 
Con respecto al efecto marginal del tratamiento (MTE) se estimó un efecto de -0.064 por ciento. El signo negativo permite demostrar que existen factores no observables (externalidades) que reducen la probabilidad de participar en el programa de ciertos individuos, quienes tienen asociado generalmente un menor rendimiento sobre sus ingresos en comparación a aquellos que decidieron llevar a cabo el programa.

En las estimaciones del efecto LATE se encontró que al ser comparadas directamente con los resultados del efecto ATE, la educación de la madre se incrementaba marginalmente. Esto además amplia la probabilidad de que el hijo decida participar en un programa de ESP. En este caso, el impacto sobre el salario del hijo se incrementa en 0.04 puntos porcentuales más, pasando de 11.2 por ciento del efecto ATE a 11.235 por ciento en el efecto local. Este resultado sugiere que la implementación de políticas públicas que incentiven la participación de un mayor número de mujeres en el ámbito educativo y/o de capacitación, adicionales a los patrones culturales ya citados por Jiménez-Villacís (2008) en este estudio, podría ser un factor detonante en la generación de mayor riqueza y de capital humano en México particularmente para las mujeres. Se encontró también que un incremento marginal en la educación del padre genera un rendimiento positivo de la ESP sobre el salario del hijo en 0.03 puntos porcentuales más, pasando de 11.2 por ciento del efecto ATE a 11.227 por ciento en el efecto local. En lo que respecta al efecto local del tamaño del hogar, se encontró, que ante un incremento marginal de éste, se tenía un impacto negativo sobre los rendimientos de la ESP del salario de los hijos en 0.08 puntos porcentuales menos, pasando de 11.2 por ciento del efecto ATE a 11.114 por ciento en el efecto local, representando una reducción esperada dado que esta variable inhibe la probabilidad de que los hijos decidan adoptar un programa de ESP.

En términos generales, los resultados empíricos obtenidos en esta investigación sugieren que el ingreso del jefe del hogar, la experiencia laboral, y variables relacionadas con el número de integrantes del hogar, entre otros factores, como la educación de los padres, influyen de manera determinante en la elección de que los individuos decidan participar en el programa de ESP, esperando con esto acceder a ingresos futuros mayores a lo largo del ciclo de vida productiva y gozar de un mayor bienestar social. Sin embargo, esta situación no es equitativa entre hombres y mujeres como se muestra a partir de los resultados encontrados, ya que las mujeres tendrían que esperar siempre rendimientos menores a lo largo de su ciclo de vida si persisten aquellos factores subyacentes que inclinan la balanza 
hacia ciertos grupos de la población. Este resultado es consistente con el encontrado por García (2008) en el ámbito latinoamericano.

Los resultados obtenidos abren la posibilidad de líneas de investigación adicionales sobre la generación de políticas públicas en México en materia de equidad de género. Un ejemplo de esto son los rendimientos esperados sobre el ingreso de las mujeres, ya que estos fueron menores, aún cuando éstas fueron más propensas a llevar a cabo el programa y contaban con niveles educativos similares en ambos grupos. De esta manera resulta ideal cuantificar el impacto de aquellas externalidades sobre el rendimiento de la educación de ciertos grupos poblacionales, pero dadas las limitaciones de información disponible en la ENIGH, no fue posible en este estudio, además de no ser el objetivo principal del mismo.

Con el objetivo de contrastar la robustez (validez interna y externa) de los resultados obtenidos, en la Tabla 8 y en la Tabla 9 se cuenta con el rendimiento privado de la Educación Superior sobre los ingresos de los individuos a partir de una submuestra.

Tabla 8: Efectos del programa de Educación Superior sobre los ingresos de los individuos

\begin{tabular}{lr}
\hline Parámetros & Valor (\%) \\
\hline Average Treatment Effect (ATE) & 10.29 \\
Marginal Treatment Effect (MTE) & -0.078 \\
Treatment on the Treated (TT) & 8.79 \\
\hline
\end{tabular}

Fuente: elaboración propia con datos de ENIGH 2012.

Tabla 9: Efecto local promedio del tratamiento de Educación Superior

\begin{tabular}{lr}
\hline Local Average Treatment Effect (LATE) & Valor (\%) \\
\hline Educación de la madre & 10.351 \\
Educación del padre & 10.352 \\
Tamaño del Hogar & 10.179 \\
\hline
\end{tabular}

Fuente: elaboración propia con datos de ENIGH 2012.

En la Tabla 8 y en la Tabla 9, en comparación con la muestra original, la conclusión más importante es que cuando se emplea una muestra más pequeña, los rendimientos fueron menores, lo cual sugiere que en la medida que la muestra empleada sea lo más robusta posible también será posible encontrar rendimientos más altos.

Al considerar los resultados mostrados en las Tablas 7 a 10, la principal implicación de comparar las dos muestras es que la metodología empleada 
y los resultados obtenidos puedieron generalizarse en función de los parámetros de interés estimados (ATE, MTE, TT y LATE), los cuales difirieron únicamente en magnitud debido al tamaño de las muestras, más no en las implicaciones demostradas en la literatura económica en este tipo de estudios.

\section{REFERENCIAS BIBLIOGRÁFICAS}

Alba-Ramírez, A. and San Segundo, M. J., 1995, "The returns to education in Spain", in Economics of Education Review, vol. 14, núm. 2. pp. 155-166.

Alonzo, R., Balisacan, A., Canlas, D., 2004, Population and poverty: the real score. Discussion Paper, núm. 0415. University of Philippines.

Altonji, J. G., 1993, "The demand for and return to education when education outcomes are uncertain", in Journal of Labor Economics, vol. 11, núm. 1, pp. 48-83.

Altonji, J. G. and Dunn, T. A., 1996, "The effects of family characteristics on the return to education", in Review of Economics and Statistics, vol. 78, núm. 4. pp. 692-704.

Angrist, J. D., Graddy, K., and Imbens, G. W., 2000, “The Interpretation of instrumental variables estimators in simultaneous equations models with an application to the demand for fish", in Review of Economic Studies, vol. 67, núm. 3, pp. 499-527.

Ariza, M., 2006, "Mercados de trabajo urbanos y desigualdad de género en México a principios del siglo XXI", en De la Garza y Salas (coords.), La situación del trabajo en México, UNAM, IET, AFL-CIO, Plaza y Valdés, pp. 377-412.

Arrazola, M., De Hevia, J., Risueño, M. and Sanz, J. F., 2003, "Returns to education in Spain: some evidence on the endogeneity of schooling", in Education Economics, vol. 11, núm. 2, pp. 293-304.

Arriagada, I., 2005, Dimensiones de la pobreza y políticas desde una perspectiva de género, Santiago de Chile, División de Desarrollo Social. Comisión Económica para América Latina y el Caribe (CEPAL), pp. 101-113.

Ashenfelter, O. and Krueger, A., 1994, "Estimates of the Economic Return to Schooling", in American Economic Review, vol. 84, núm. 5, pp. 1157-1173.

Asplund, R. and Pereira, P. T., 1999, Returns to human capital in Europe. A literature review, Helsinki: ETLA.

Austria, C. M. y Venegas-Martínez, F., 2010, "Rendimientos privados de la Educación Superior en México en 2006 un modelo de corrección del sesgo por autoselección", in El Trimestre Económico, vol. 78(2), núm. 310, pp. 441-468.

Baker, J. L., 2000, "Evaluación del impacto de los proyectos de desarrollo en la pobreza", en Manual para Profesionales. Banco Mundial. Washington, D. C. 
Barceinas, F., 2001, Capital humano y rendimientos de la educación en México, Tesis Doctoral, Universidad Autónoma de Barcelona.

Becker, G. S., 1962, "Investment in human capital: a theoretical analysis", in Journal of Political Economy, vol. 70. núm. 5, pp. 9-49.

Becker, G. S., 1964, Human capital: a theoretical and empirical analysis, with special reference to education, National Bureau of Economic Research, New York.

Bracho, T. y Zamudio, A., 1994a, Rendimientos económicos a la escolaridad I: discusión teórica y métodos de estimación. Documento de Trabajo 30. CIDE. México.

Bracho, T. y Zamudio, A., 1994b, Rendimientos económicos a la escolaridad II: estimaciones para el caso mexicano. Documento de Trabajo 31. CIDE. México

Campbell, D. T. y Stanley, J. C., 1973, Diseños experimentales y cuasi-experimentales en la investigación social. Buenos Aires: Amorrortu.

Cabo, S. G. y Garzón, M. J., 2007, Diferencia y discriminación salarial por razón de sexo. Instituto de la Mujer (MTAS). Centro de Estudios Económicos Tomillo, S. L. Madrid.

Card, D., 1999, "The causal effect of education on earnings", en Ashenfelter, O. y Card, D. (eds.), Handbook of Labor Economics, vol. 3, North-Holland, Amsterdam.

Card, D., 2000, Estimating the return to schooling: progress on some persistent econometric problems. Working Paper 7769, NBER.

Carneiro, P., J. Heckman and Vytlacil, E., 2001, Estimating the returns to education when it varies among individuals, Working Paper. University of Chicago.

Carneiro, P., K. Hansen and Heckman, J., 2003, Estimating distributions of treatment effects with an application to the returns to schooling and measurement of the effects of uncertainty of college choice. NBER Working Paper, $\mathrm{N}^{\circ} 9546$.

Carnoy, M., 1967, "Earnings and Schooling in Mexico", in Economic Development and Cultural Change, vol. 15, núm. 4. pp. 408-418.

Cohn, E., and Addison, J. T., 1998, "The economics returns to lifelong learning in OECD countries", in Education Economics, vol. 6, núm. 3, pp. 253-307.

CEPAL, 2004, Caminos hacia la equidad de género en América Latina y el Caribe, documento presentado a la $9^{a}$ Conferencia Regional sobre la Mujer de América Latina y el Caribe, México, D. F., 10 al 12/06/2004.

CEPAL/OREALC, 1992, Educación y conocimiento: eje de la transformación productiva con equidad, Comisión Económica para América Latina y el Caribe/ Oficina Regional de Educación de la UNESCO para América Latina y el Caribe. Santiago de Chile. Publicación de las Naciones Unidas.

CEPAL-UNI FEM, 2004, Entender la pobreza desde la perspectiva de género. Serie mujer y desarrollo, (52). Chile.

Consejo Nacional de Evaluación de la Política de Desarrollo Social, 2012, Pobreza y Género en México: Hacia un sistema de indicadores información 2008-2012. 
Del Razo, L. M., 2003, Estudio de la brecha salarial entre hombres y mujeres 1994-2001. Serie Documentos de Investigación. Secretaría de Desarrollo Social. 2003.

Diez de Medina, R., 1992, "El sesgo de selección en la actividad de jóvenes y mujeres", in Suma. vol. 7, núm. 13, pp. 69-85.

ENIGH, 2012, Encuesta Nacional de Ingresos y Gastos de los Hogares de 2012. Instituto Nacional de Estadística Geografía e Informática (INEGI). Disponible en http://www.beta.inegi.org.mx/proyectos/enchogares/regulares/enigh/tradicio$\mathrm{nal} / 2012 /$

Faúndez, A., 1996, Género, salud y políticas públicas: del binomio madre-hijo a la mujer integral. Memoria para optar al título de Magister en Gestión y Políticas Públicas, Santiago de Chile. Universidad de Chile.

García, E., 2008, "Políticas de igualdad, equidad y gender mainstreaming." Programa de las Naciones Unidas para el Desarrollo (PNUD). América Latina Genera: Gestión del Conocimiento para la Equidad de Género en Latinoamérica y el Caribe.

García, B. y de Oliveira, O., 2007, “Trabajo extradoméstico y relaciones de género: una nueva mirada", en Género, familias y trabajo: rupturas y continuidades. Desafios para la investigación política. CLACSO, Buenos Aires.

García de Fanelli, A. M., 1989, "Patrones de desigualdad social en la sociedad moderna: una revisión de la literatura sobre discriminación ocupacional y salarial por género", in Revista Desarrollo Económico, Vol. 29, No. 114, pp. 239-264.

Gerstenfeld, P. y León, A., 1999, Transmisión intergeneracional de la desigualdad. Santiago de Chile, Comisión Económica para América Latina y el Caribe (CEPAL), inédito.

Goldschmidt-Clermont, L., 1995, "La valoración monetaria del trabajo no remunerado”, in Revista Política y Sociedad, núm. 19, mayo-agosto, pp. 7-18.

Gómez, E., 2002, “Género, equidad y acceso a los servicios de salud: una aproximación empírica”, en Revista Panamericana de Salud Pública, vol. 11 núm. 5/6, pp. 327-334.

Hanoch, G., 1967, “An economic analysis of earnings and schooling”, in Journal of Human Resources, vol. 2, núm. 3, pp. 310-329.

Hansen, W. L., 1963, “Total and private rates of return to investment in schooling”, in Journal of Political Economy, vol. 71, núm. 2, pp. 128-140.

Harmon, C., H. Oosterbeek, and Walter, I., 2003, "The returns to education: microeconomics", in Journal of Economic Surveys, vol. 17, núm. 2, pp. 115-155.

Harmon, C., I. Walker and Westergaard-Nielsen, N., 2001, Education and earnings in Europe. A cross country analysis of the returns to education, Cheltenham: Edward Elgar. 
Harmon, C., and Walter, I., 1995, "Estimates of the economic return to schooling for the United Kingdom", in American Economic Review, vol. 85, núm. 5, pp. 1278-1286.

Heckman, J., 1997, "Instrumental variables: a study of the implicit assumptions underlying one widely used estimator for program evaluation", in Journal of Human Resources, bol. 32, núm. 3, pp. 441-462.

Heckman, J. and Vytlacil, E., 2000, Identifying the role of cognitive ability in explaining the level of and change in the return to schooling, Working Paper 7820, Cambridge, Mass. NBER.

Heckman, J., Tobias, J. L. and Vytlacil, E., 2000, Simple estimators for treatment parameters in a latent variable framework with an application to estimating the returns to schooling, Working Paper 7950, NBER.

Heckman, J., J. L. Tobias and E. Vytlacil, 2001, "Four parameters of interest in the evaluation of social programs", in Southern Economic Journal, vol. 68, núm. 2, pp. 210-223.

Heckman, J., Tobias, J. L. and Vytlacil, E., 2005, Structural equations, treatment effects and econometric policy evaluation, Working Paper 306, NBER.

Imbens, G. and Angrist , J., 1994, "Identification and estimation of local average treatment effects, in Econometrica", vol. 62, núm. 2, pp. 467-475.

Jiménez-Villacís, M., 2008, Influencia de los padres en el rendimiento escolar de sus hijos. Universidad del Mayab.

Katrin, E., Newiak, M., Kochhar, K., Fabrizio, S., Kpodar, K., Wingender, P. Clements, B. y Schwartz, G., 2013, Las mujeres, el trabajo y la economía: Beneficios macroeconómicos de la equidad de género. Departamento de Estrategia, Políticas y Evaluación y Departamento de Finanzas Públicas. Fondo Monetario Internacional.

López-Acevedo, G., 2004, "Mexico: Evolution of earnings inequality and rates of returns to education (1988-2002)", in Estudios Económicos, vol. 19, núm. 2, pp. 211-284.

McMahon, W. W. E., 1991, "Relative returns to human and physical capital in the U. S. and efficient investment strategies", in Economics of Education Review, vol. 10, núm. 4, pp. 283-296.

Mincer, J., 1974, Schooling, experience and earnings, New York. National Bureau of Economic Research.

Moffitt, R., 2007, Estimating marginal returns to higher education in The UK. National Bureau of Economic Research. NBER Working Paper No. 13534.

Mroz, T. A., 1987, “The sensitiviy of an empirical model of married women's hours of work to economic and statistical assumptions", in Econometrica, vol. 55, núm. pp. 765-799.

Ordaz, J. L., 2007, México: capital humano e ingresos. Retornos a la educación, 1994-2005. CEPAL-Serie Estudios y Perspectivas, núm. 90, octubre, pp. 1-69. 
Parker, S. W. and Pederzini, C. 2000, "Género y educación en México", en Estudios Demográficos y Urbanos, vol. 15, núm. 1, pp. 97-122.

Psacharopoulos, G., 1993, Returns to investment in education: a global update, Policy Research Working Paper, núm. 1067. Banco Mundial.

Psacharopoulos, G. and Patrinos, H., 2002, Returns to investment in education: a further update, Policy Research Working Paper, núm. 2881, Banco Mundial.

Raczynski, D., 2003, Equidad, inversión social y pobreza. Innovar en cómo se concibe, diseña y gestiona las políticas y los programas sociales. Documento presentado en el Seminario CEPAL/MIDEPLAN "Políticas innovativas de desarrollo social". Santiago de Chile, 23 y 24 de mayo.

Rodríguez-Oreggia, E., 2004, Institutions, geography and the regional evolution of returns to schooling in México. Instituto de Investigaciones sobre Desarrollo Sustentable y Equidad Social, Universidad Iberoamericana-Santa Fe, México, inédito.

Rojas M., H. Angulo, e I. Velásquez, 2000, "Rentabilidad de la inversión en capital humano en México”, en Economía Mexicana, vol. 9, núm. 2, pp. 113-142.

Salles, V. y Tuirán, R., 2002, ¿Cargan las mujeres con el peso de la pobreza? Puntos de vista de un debate. Guatemala. Sistema de Naciones Unidas.

San Segundo, M. J., and Valiente, A., 2003, "Family background and returns to schooling in Spain", in Education Economics, vol. 11, núm. 1, pp. 39-52.

Sarimaña, J. E., 2002, "Rendimiento de la escolaridad en México: Una aplicación del método de variables instrumentales para 1998", en Gaceta de Economía, vol. 7, núm. 14, pp. 85-125.

Schultz, T. W., 1961, "Investment in Human Capital”, in American Economic Review, vol. 51, núm. 1, pp. 1-17.

Skipper, S., 2006, "Desarrollo del capital humano y reducción de la pobreza en el Salvador”, en Comercio Exterior, vol. 56, núm. 11, pp. 968-976.

Wagstaff, A., 2002, "Pobreza y desigualdades en el sector de la salud", en Revista Panamericana de Salud, vol. 11, núm. 5/6, pp. 316-326.

Willms, J. D., 2006, Learning divides: ten policy questions about the performance and equity of schools and schooling systems. Montreal: UNESCO.

\section{RESUMEN CURRICULAR DE LOS AUTORES}

\section{Marco Antonio Austria Carlos}

Doctor en Economía por el Instituto Politécnico Nacional. Investigador responsable de Proyecciones y Prospectiva Económica en el Centro de Estudios de las Finanzas Públicas del H. Congreso de la Unión, desde enero de 2015 a la fecha; consultor independiente desde 2004, en donde ha parti- 
cipado en la evaluación de programas de sociales a cargo de la SEDESOL e IMSS. Entre sus publicaciones más importantes destaca (en coautoría con el Dr. Francisco Venegas-Martínez) Rendimientos privados de la educación superior en México en 2006. Un modelo de corrección del sesgo por autoselección (2011). El Trimestre Económico.

Dirección electrónica: autriche357@gmail.com

\section{Francisco Venegas Martínez}

Doctor en Matemáticas y Doctor en Economía por Washington Sate University. Es Profesor-Investigador en la Escuela Superior de Economía del Instituto Politécnico Nacional desde 2006. Ha sido galardonado con la entrega de la Presea Lázaro Cárdenas del Río por el Presidente de la República Mexicana. Es miembro del Sistema Nacional de Investigadores nivel III. Entre sus publicaciones más importantes destaca Riesgos Financieros y Económicos: Productos derivados y decisiones económicas bajo incertidumbre.

Dirección electrónica: fvenegas1111@yahoo.com.mx

\section{Gilberto Pérez Lechuga}

Doctor en Ingeniería (Investigación de Operaciones) por la Universidad Nacional Autónoma de México. Ha sido Director de la Escuela Superior de Apan de la Universidad Autónoma del Estado de Hidalgo, 2010 a 2017. Es miembro del Consejo Asesor Internacional del International Journal of Energy Optimization \& Engineering. Es Director General y fundador de SEIIO, Consultora Internacional en Ingeniería Industrial e Investigación Operativa. Actualmente es Director de Investigación de la Universidad Autónoma del Estado de Hidalgo. Es miembro del Sistema Nacional de Investigadores. Entre sus publicaciones más importantes destaca Optimal Logistics Strategy to Distribute Medicines in Clinics and Hospitals (2018). Journal of Mathematics in Industry.

Dirección electrónica: glechuga@uaeh.edu.mx

Artículo recibido el 17 de agosto de 2015 y aprobado el 4 de septiembre de 2017. 